\title{
Magnetic and transport properties of $\mathrm{La}_{0.7} \mathrm{Sr}_{0.3} \mathrm{MnO}_{3} / \mathrm{Pr}_{0.65} \mathrm{Ca}_{0.35} \mathrm{MnO}_{3}$ multilayered films with different microstructure
}

\author{
V.G. Prokhorov, V.S. Flis, and G.G. Kaminsky \\ Institute of Metal Physics, National Academy of Sciences of Ukraine \\ 36 Vernadskogo Ave., Kiev, 03142, Ukraine \\ Email: pvg@imp.kiev.ua \\ Y.P. Lee \\ Quantum Photonic Science Research Center and Department of Physics \\ Hanyang University, Seoul, 133-791 Korea
}

Received October 29, 2003, revised December 19, 2003

\begin{abstract}
The magnetic and transport properties of single-crystal and polycrystalline $\mathrm{La}_{0.7} \mathrm{Sr}_{0.3} \mathrm{MnO}_{3} / \mathrm{Pr}_{0.65} \mathrm{Ca}_{0.35} \mathrm{MnO}_{3}$ multilayered films are investigated in the temperature range 4.2-300 K. It is shown that the transformation from an incoherent to a coherent interface between layers leads to an enhancement of the ferromagnetic coupling, which is accompanied by a modification in the temperature dependence of the resistance and by a grown negative magnetoresistance ratio at room temperature. The influence of grain boundaries on the transport of carriers in the multilayered films is discussed on the basis of modern theoretical approaches.
\end{abstract}

PACS: 71.30.+ h, 75.47.Gk, 75.47.Lx

The hole-doped perovskite manganites, $\mathrm{R}_{1-x} \mathrm{~A}_{x} \mathrm{MnO}_{3}(\mathrm{R}=$ rare-earth cation, $\mathrm{A}=$ alkali or alkaline-earth cation), have attracted considerable attention due to not only their interesting fundamental science, partly connected with the discovery of colossal magnetoresistance (CMR), but their potential for device applications [1,2]. Most of the early theoretical works on manganites focused on the relation between the transport and magnetic properties and explained the coexistence of ferromagnetism and metallic behavior within the framework of a «double exchange» (DE) model, which considers the magnetic coupling between $\mathrm{Mn}^{3+}$ and $\mathrm{Mn}^{4+}$ that results from the motion of an electron between two partially filled $d$ shells with strong on-site Hund's coupling [3-5]. In spite of considerable scientific efforts, however, the complex interplay between the charge, lattice, spin, and orbital degrees of freedom in these systems is not completely understood. The situation is further complicated by the fact that the magnetic and transport properties are dependent significantly on the cation size, the lattice strain, and the microstructure. Recently it was found that the presence of grain boundaries (GBs) in the polycrystalline manganites leads to a large MR effect over a wide temperature range below the Curie temperature $T_{C}$, whereas the CMR in the single-crystal materials is restricted to a narrower temperature range just around $T_{C}[6-8]$.

The most widely different manganite compounds were chosen for fabrication of the multilayer structure in this work: $\operatorname{Pr}_{0.65} \mathrm{Ca}_{0.35} \mathrm{MnO}_{3}$ (PCMO) and $\mathrm{La}_{0.7} \mathrm{Sr}_{0.3} \mathrm{MnO}_{3}$ (LSMO). The first of them remains insulator in both the paramagnetic and ferromagnetic states [9] (or displays an incomplete metal-insulator (MI) transition in the lattice-strained state [10]), and the second one shows a metal-like behavior of electrical resistance in the whole temperature range [11]. In spite of the fact that $T_{C}$ of LSMO exceeds room temperature, this compound manifests insignificant changes in resistance in applied magnetic fields owing to a small value of the intrinsic resistivity in the metallic state. It can be expected that the presence of high-resistance PCMO layers can lead to an enhancement of the magnetoresistance effect in the 
$\mathrm{PCMO} / \mathrm{LSMO}$ multilayered films. Recently it was reported that the substitution of the small-size $\mathrm{Pr}$ ion by La in the compound $\mathrm{Pr}_{0.67} \mathrm{Ca}_{0.33} \mathrm{MnO}_{3}$ leads to the appearance of a MI transition at low temperature owing to the melting of a charge-ordered insulating state [12]. On the other hand, the substitution of $\mathrm{Sr}$ for $\mathrm{Ca}$ in $\mathrm{Pr}_{0.7} \mathrm{Ca}_{0.3-x} \mathrm{Sr}_{x} \mathrm{MnO}_{3}$ induces the formation of a low-temperature metallic state, as well [13].

In this paper we report experimental results concerning the magnetic and transport properties of $\mathrm{Pr}_{0.65} \mathrm{Ca}_{0.35} \mathrm{MnO}_{3} / \mathrm{La}_{0.7} \mathrm{Sr}_{0.3} \mathrm{MnO}_{3}$ (PCMO/LSMO) multilayered (ML) films prepared by laser ablation on single-crystal $\mathrm{LaAlO}_{3}(\mathrm{SC})$ and polycrystalline $\mathrm{Al}_{2} \mathrm{O}_{3}$ (PC) substrates at two different temperatures, $T_{\text {sub }}=$ $=560$ and $710{ }^{\circ} \mathrm{C}$. The low $T_{\text {sub }}$ were used for preparation of the ML films to avoid chemical interaction between layers. In the first case, high-textured ML films (which will be denoted by SC) were fabricated with a coherent and an incoherent interface between layers, which was controlled by $T_{\text {sub }}$. In the second case, polycrystalline ML films were obtained. It was shown that the transformation from an incoherent to a coherent interface between layers leads to an enhancement of the ferromagnetic coupling in the SCML films. This process is accompanied by a modification in the temperature dependence of resistance from $R \propto T^{3}$ to $\propto T^{4.5}$ and has been attributed to transition from one- to two-magnon-electron scattering. The PCML films demonstrate the $R \propto T^{2}$ behavior, which is explained by the interference between the elastic electron scattering on GBs and the electron-magnon scattering. The exponential growth of resistance at low temperature, $R(T) \propto \exp \sqrt{E_{C} / T}$, has its origin in a small Coulomb barrier which formed on the GBs. The MR ratio of the PCML films is dominated by a spin-polarized tunneling between grains. It was shown that the model of two parallel resistances can be used for a simulation of the transport properties in the ML films.

\section{Experimental details}

A pulsed-laser-deposition method was employed for the preparation of the films. We used two Nd-YAG lasers with a wavelength of $1064 \mathrm{~nm}$, a pulse duration of $7.8-10.5 \mathrm{~ns}$, and an energy of $0.3 \mathrm{~J} /$ pulse. The film deposition was carried out at a pulse repetition rate of $20 \mathrm{~Hz}$. The power density of a laser beam focused on the target was $9.5 \cdot 10^{8}-2 \cdot 10^{10} \mathrm{~W} / \mathrm{cm}^{2}$. The targets were fabricated from the $\mathrm{Pr}_{0.65} \mathrm{Ca}_{0.35} \mathrm{MnO}_{3}$ and $\mathrm{La}_{0.7} \mathrm{Sr}_{0.3} \mathrm{MnO}_{3}$ powders of the stoichiometric composition by hot-pressing and heating at $1200^{\circ} \mathrm{C}$ for 4 days in air. The oxygen pressure in chamber was 200 Torr during deposition and 600 Torr during cooling. Under these conditions we grew the ML films on
$\mathrm{LaAlO}_{3}(100)$ single-crystal and $\mathrm{Al}_{2} \mathrm{O}_{3}$ polycrystalline substrates at different temperatures, $T_{\text {sub }}=560{ }^{\circ} \mathrm{C}$ and $710^{\circ} \mathrm{C}$. The ML films contain six LSMO and five PCMO layers with LSMO at the top and the bottom. The thickness of each layer was $\simeq 20 \mathrm{~nm}$.

The $\theta-2 \theta$ x-ray diffraction (XRD) patterns were obtained using a Rigaku diffractometer with $\mathrm{Cu} K_{\alpha 1}$ radiation. The lattice parameters evaluated directly from the XRD data were plotted against $\cos ^{2} \theta / \sin \theta$. With an extrapolated straight line to $\cos ^{2} \theta / \sin \theta=0$, a more precise determination of the lattice parameter was obtained. The resistance measurements were performed by using the standart four-probe method. The temperature dependence of the field-cooled (FC) and the zero-field-cooled (ZFC) in-plane magnetization at a magnetic field of 100 Oe was taken with a Quantum Design SQUID magnetometer.

\section{Results and discussion}

\section{Structure}

Figure 1, $a$ presents the $\theta-2 \theta$ XRD scans for two ML films deposited on $\mathrm{LaAlO}_{3}$ at $T_{\text {sub }}=560{ }^{\circ} \mathrm{C}$ (SCML1) and $710^{\circ} \mathrm{C}$ (SCML2). SCML1 and SCML2 are the multilayred film deposited on the single-crystal $\mathrm{LaAlO}_{3}$ substrate at $560{ }^{\circ} \mathrm{C}$ and $710{ }^{\circ} \mathrm{C}$, respectively. High intensities of the $(00 l)$ peaks attest that the deposition results in highly $c$-oriented films.
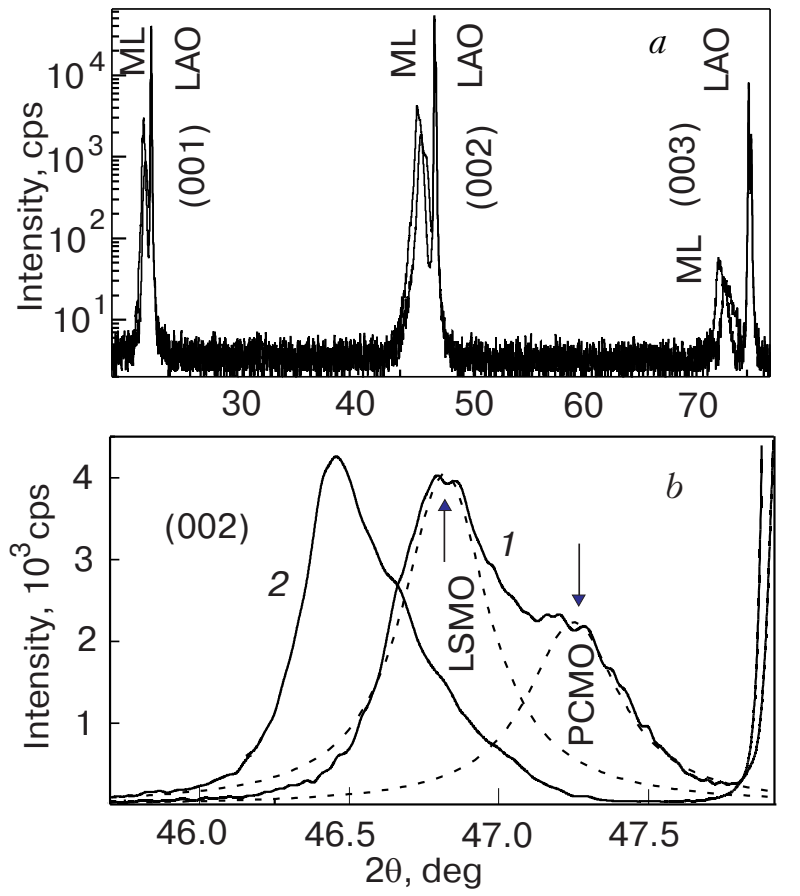

Fig. 1. (a) XRD patterns of the SCML films. LAO and ML denote the diffraction peaks from the substrate and the film itself, respectively. (b) The (002) diffraction peaks for the SCML1 (1) and the SCML2 (2) films. 
Figure 1, $b$ is the (002) Bragg peaks for the SCML1 (curve 1) and SCML2 (curve 2) films, respectively. It is seen that the decrease in $T_{\text {sub }}$ leads to a shift of the Bragg peak towards a larger angle. In addition to the decrease in out-of-plane lattice parameter, the SCML1 film displays a kink-like peculiarity in the (002) peak, which can be interpreted as the presence of different crystalline phases with the following out-of-plane lattice parameters: $c \simeq 0.3877$ and $0.3848 \mathrm{~nm}$. To show this more clearly, Fig. $1, b$ includes a fitting to the split (002) peak by using two Lorentzian functions (dashed lines). The first lattice parameter is almost coincident with that for the bulk LSMO single crystal, $a_{c}=0.3876 \mathrm{~nm}$ [11], while the second one is similar to the average lattice parameter of the bulk PCMO compound, $\left\langle a_{c}\right\rangle \simeq 0.3843 \mathrm{~nm}$ [14], for the cubic symmetry. The SCML2 film displays only the unsplit Bragg peak which corresponds to $c \simeq 0.3903 \mathrm{~nm}$. Therefore, one can conclude that at a low $T_{\text {sub }}$ the PCMO and the LSMO layers form an incoherent interface and have different lattice parameters close to those of the respective bulk materials. An increase in $T_{\text {sub }}$ provides an enhancement of the epitaxial growth process and induces the formation of a coherent interface between layers in the SCML2 film. The ML films deposited on polycrystalline $\mathrm{Al}_{2} \mathrm{O}_{3}$ (PCML) exhibit the multipeak XRD patterns of very weak intensity, which are beyond an analysis.

\section{Magnetic properties}

Figures 2, $a$ and 2,b present the temperature dependence of $\mathrm{FC}$ and ZFC magnetization, $M(T)$, for SCML1 (curve 1) and SCML2 (curve 2), and PCML1 (curve 1) and PCML2 (curve 2), respectively. PCML1 and PCML2 are the multilayered films deposited on the polycrystalline $\mathrm{Al}_{2} \mathrm{O}_{3}$ substrate at $T_{\text {sub }}=560{ }^{\circ} \mathrm{C}$ and $710^{\circ} \mathrm{C}$, respectively. The SCML1 film (curve 1 in Fig. 2,a) demonstrates an $M(T)$ dependence, which is typical for two-phase magnetic systems, and represents a superposition of two magnetic transitions for the PCMO layers at $T_{C 2} \approx 130 \mathrm{~K}[10]$ and for the LSMO layers at $T_{C 1}>300 \mathrm{~K}$ [15]. Moreover, the absolute value of the magnetization at low temperatures is almost twice greater than that at $T>T_{C 2}$. This is evidence for independent magnetic transitions in six LSMO and in five PCMO layers, and for a lack of ferromagnetic coupling between them. We are claiming that the main reason for a suppression of the magnetic coupling between layers in this film is the aforementioned incoherence of their interfaces. The increase of $T_{\text {sub }}$ leads to the conversion into a coherent interface and thus to the appearance of a ferromagnetic coupling between two kinds of layers. The SCML2 film displays a monotonic $M(T)$ dependence (curve 2 in Fig. 2,a)

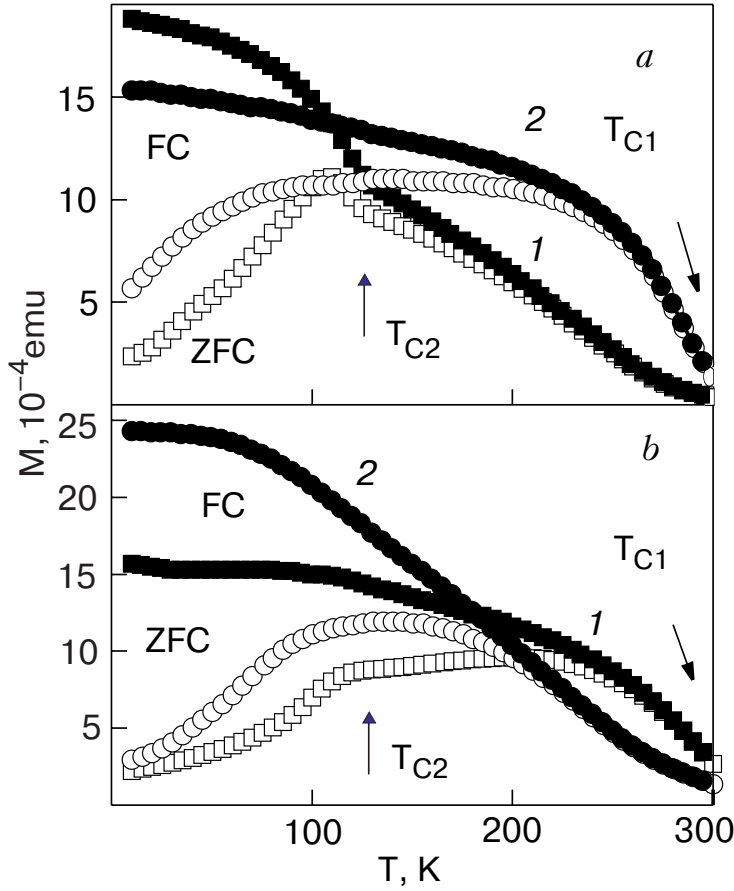

Fig. 2. (a) Temperature dependence of FC (solid) and ZFC (open) magnetization for the SCML1 (1) and the SCML2 (2) films. (b) The same for the PCLM1 (1) and the PCML2 (2) films.

without any peculiarity at $T_{C 2}$ which is relevant to the magnetic transition for the PCMO layers. Figure 2, $b$ shows that a similar transformation in the magnetic state governed by the substrate temperature is also typical for the PCML films. The PCML1 film manifests a kink-like peculiarity at $T_{\mathrm{C} 2}$ on both $\mathrm{FC}$ and ZFC $M(T)$ curves (curve 1 in Fig. 2,b), although the magnitude is greatly smaller than that observed for the SCML1 film. The increase of $T_{\text {sub }}$ leads to a degradation of the peculiarity (curve 2 in Fig. 2,b). A similar ferromagnetic coupling was observed recently in $\mathrm{La}_{0.55} \mathrm{Sr}_{0.45} \mathrm{MnO}_{3} / \mathrm{La}_{0.9} \mathrm{Sr}_{0.1} \mathrm{MnO}_{3}\left(\mathrm{La}_{0.67} \mathrm{Ca}_{0.33} \mathrm{MnO}_{3}\right)$ multilayered films prepared at a high temperature of substrate [16].

Therefore, one can conclude that the ferromagnetic coupling between layers in multilayered films is controlled by the coherence ratio of their interfaces.

\section{Transport properties}

Figure 3 shows the temperature dependence of resistance $R(T)$ for the SCML1 (curve 1) and the SCML2 (curve 2) films without (solid symbols) and with (open symbols) an applied magnetic field of $5 \mathrm{~T}$. The magnetic field was parallel to the film surface and normal to the transport current. The change in the magnetic field direction does not transform the $R(T)$ behavior. The experimental curves testify that the resistance peak $T_{P}$ in the investigated temperature range is observed only for the SCML1 film (curve 1). A similar tempera- 


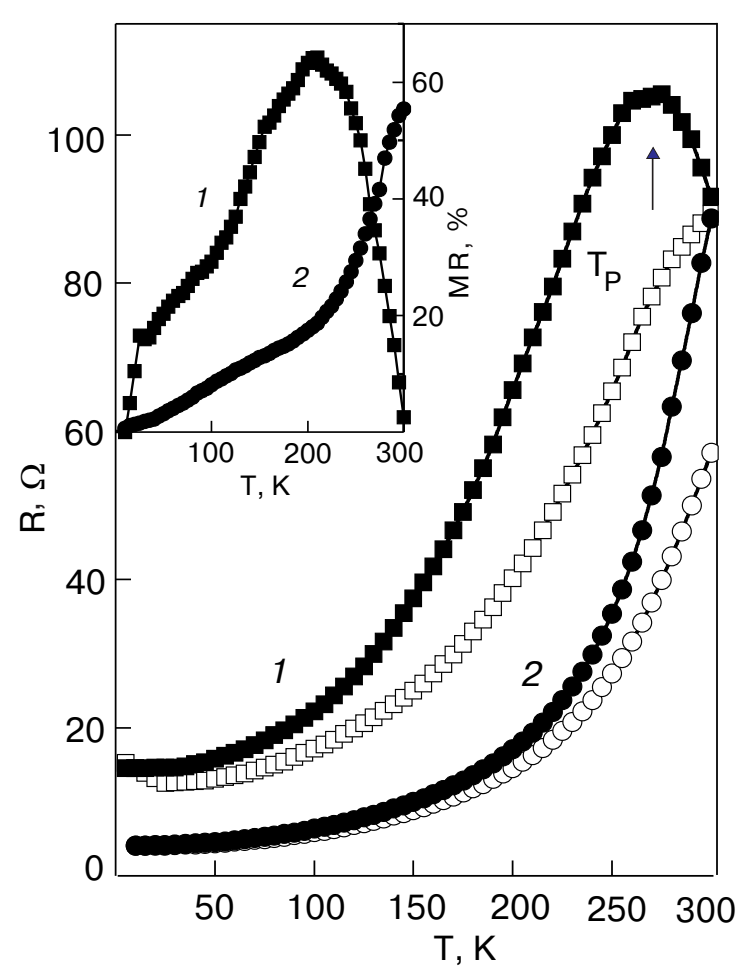

Fig. 3. Temperature-dependent resistance of the SCML1 (1) and SCML2 (2) films, measured in a magnetic field of zero (solid) and $5 \mathrm{~T}$ (open). The inset displays the MR ratio in a perpendicular magnetic field of $5 \mathrm{~T}$. The solid lines are drawn to guide the eye.

ture behavior of resistance was observed recently for $\mathrm{La}_{2 / 3} \mathrm{Ca}_{1 / 3} \mathrm{MnO}_{3} / \mathrm{Pr}_{2 / 3} \mathrm{Ca}_{1 / 3} \mathrm{MnO}_{3}$ multilayered films with a sublayer thickness of $10 \mathrm{~nm}$, but at a lower temperature [17]. The SCML2 film (curve 2) demonstrates a metallic-like behavior of the $R(T)$ in the whole investigated temperature range. Inset in Fig. 3 shows the temperature dependence of the magnetoresistance ratio. The MR value is defined by $100 \% \times[R(0)-$ $-R(H) / R(0)$, where $R(0)$ and $R(H)$ are the resistance with and without a magnetic field of $5 \mathrm{~T}$, respectively. It is seen that for SCML1 (curve 1) $\operatorname{MR}(T)$ shows the nonmonotonic dependence with a peak at $T \simeq 200 \mathrm{~K}$, while the $\mathrm{MR}(T)$ of SCML2 increases monotonically with temperature and reaches almost $60 \%$ at room temperature, which is much greater than that ever observed for the bare LSMO film [18].

Figure 4 displays the temperature dependence of resistance for the PCML1 (curve 1) and the PCML2 (curve 2) films without (solid symbols) and with (open symbols) an applied magnetic field of $5 \mathrm{~T}$. It is seen that the $R(T)$ behavior of the PCML films differs significantly from that of the SCML ones. Inset in Fig. 4 shows that in contrast to the single-crystal ML films the MR value for the PCML films is minimum at room temperature and increases with decreasing temperature.

Single-crystal multilayered films. First of all, let us consider the $R(T)$ behavior of SCML films. Figure 3

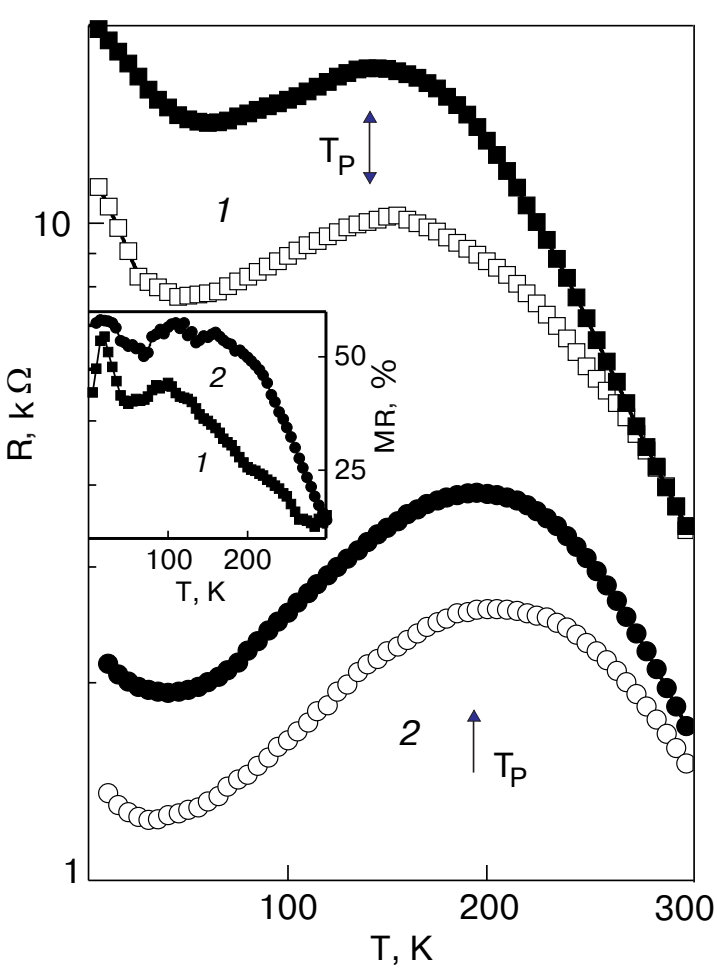

Fig. 4. Temperature-dependent resistance of the PCML1 (1) and the PCML2 (2) films, measured in a magnetic field of zero (solid) and $5 \mathrm{~T}$ (open). The inset displays the $\mathrm{MR}$ ratio in a perpendicular magnetic field of $5 \mathrm{~T}$. The solid lines are drawn to guide the eye.

(curve 1) shows that the MI transition in the SCML1 film $\left(T_{P} \simeq 260 \mathrm{~K}\right)$ occurs at a temperature below the Curie point $\left(T_{C} \gtrsim 300 \mathrm{~K}\right)$. As a rule, the temperature difference between the magnetic and electronic transitions in CMR compounds is explained by an intrinsic inhomogeneity of these materials and by a percolative nature of the conductivity [19]. On the other hand, a more simplified explanation can be employed in our case. Recently the two parallel resistor model was used for description of the transport properties of bi- and trilayers $\mathrm{La}_{0.55} \mathrm{Sr}_{0.45} \mathrm{MnO}_{3} / \mathrm{La}_{0.67} \mathrm{Sr}_{0.33} \mathrm{MnO}_{3}$ films [20]. The equivalent resistance for the SCML film can be described by $R^{-1}(T)=R_{\mathrm{PCMO}}^{-1}(T)+R_{\mathrm{LSMO}}^{-1}(T)$, where $R_{\mathrm{PCMO}}(T)$ and $R_{\mathrm{LSMO}}(T)$ are the total resistances of the PCMO and the LSMO layers, respectively. Therefore, the resistance of the multilayered film is determined by the electron transport in PCMO at high temperature $\left(R_{\mathrm{PCMO}}<<R_{\mathrm{LSMO}}\right)$ or in LSMO at low temperature $\left(R_{\mathrm{LSMO}} \ll R_{\mathrm{PCMO}}\right)$. The $R_{\mathrm{PCMO}}(T)$ behavior can be approximated by the usual Arrhenius form, which is typical for the bare PCMO films [10] and is provided by a polaron motion [2,21]: $R_{\mathrm{PCMO}}(T)=R_{0} T \exp \left(T_{A} / T\right)$, where $T_{A}$ is the activation energy in units of temperature. The $R_{\mathrm{LSMO}}(T)$ behavior can be obtained directly from the low-temperature part of the experimental $R(T)$ dependence. The inset in Fig. 5 shows that for the LSMO layers, $R_{\mathrm{LSMO}}(T)=R_{1}+\alpha T^{3}$ for SCML1 and 


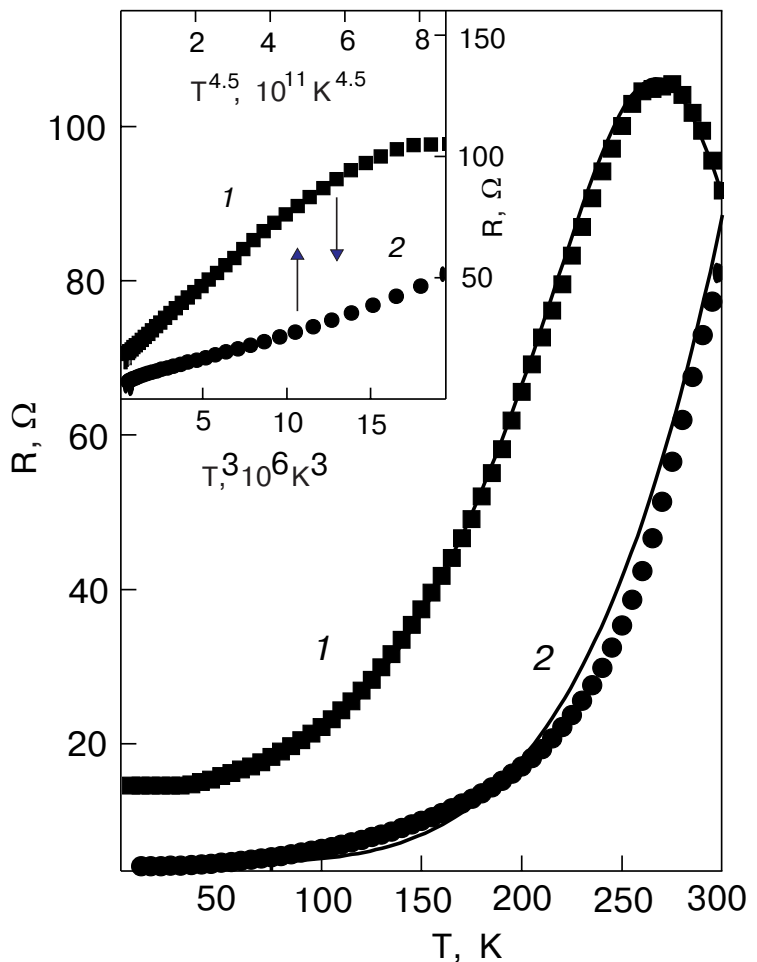

Fig. 5. The comparison of the experimental $R(T)$ data (solid symbols) with the theoretical simulation (solid lines) in the framework of the two-parallel resistance model for the SCML1 (1) and the SCML2 (2) films. The inset displays plots: $R$ versus $T^{3}$ and $R$ versus $T^{4.5}$ for the same films.

$R_{\mathrm{LSMO}}(T)=R_{2}+\beta T^{4.5}$ for SCML2 with the following fitting parameters: $R_{1}=15 \Omega, \alpha=6.5 \cdot 10^{-6} \Omega \cdot \mathrm{K}^{-3}$, $R_{2}=4.5 \Omega$, and $\beta=6 \cdot 10^{-10} \Omega \cdot \mathrm{K}^{-4.5}$. According to theoretical models, the $T^{3}$ term in the resistance corresponds to the one-magnon-electron scattering [22], while the $T^{4.5}$ term reflects the two-magnon-electron scattering processes [23]. Therefore, the electron-magnon scattering is dominating for the LSMO layers in the SCML films and transforms from one- to two-magnon scattering with an enhancement of the ferromagnetic coupling between layers. The solid lines in Fig. 5 display the theoretical $R(T)$ curves, which were calculated in the framework of the two parallel resistor model with the use of the previous fitting parameters for the LSMO layers, and $R_{0} \simeq 10^{-3} \Omega \cdot \mathrm{K}^{-1}$ and $T_{\mathrm{A}}=2600 \mathrm{~K}$ for the PCMO layers. It is seen that the theoretical curves agree excellently with the experimental data.

Polycrystalline multilayered films. Figure 4 shows that the $R(T)$ behavior for the PCML films is very different from that for the SCML ones, exhibiting a wide maximum at temperature well below $T_{C}$ and a minimum at $T_{\min } \simeq 30-40 \mathrm{~K}$. The temperature dependence of the MR ratio is very close to that for ferromagnetic tunnel junctions [24]. The similar peculiarities in the transport properties are typical for the
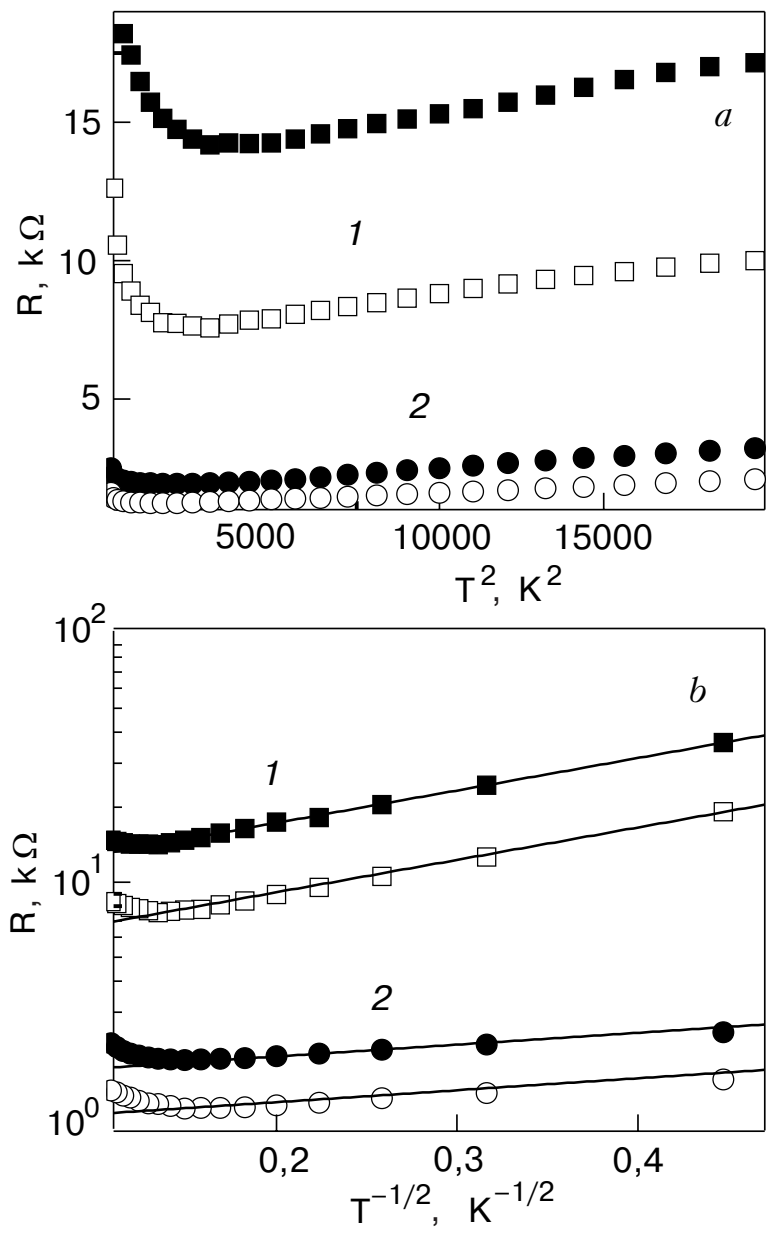

Fig. 6. The $R$ versus $T^{2}(a)$ and $\ln (R)$ versus $T^{-1 / 2}(b)$ plots for the PCML1 (1) and the PCML2 (2) films, respectively.

polycrystalline doped manganites and are explained by the presence of the grain boundaries $[2,8,25,26]$.

The GBs can play two roles in the mechanism of the transport of carriers - first as a network of magnetic tunnel junctions and second as additional centers for the elastic electron scattering in a metal-like channel of conductivity. First of all, let us consider the $R(T)$ behavior of the PCML films in the metal-like state $\left(T_{\min } \leq T<T_{P}\right)$ and discuss the influence of GBs on the transport properties. Figure 6, $a$ shows that in this temperature range $R(T) \propto T^{2}$ for both of the polycrystalline multilayered films instead of $T^{3}$ and $T^{4.5}$, which were observed for the single-crystal ones (see the inset in Fig. 5). Moreover, the applied magnetic field does not change the $R(T)$ behavior fundamentally but only decreases the slope of the curves slightly. In numerous publications the square term in the temperature-dependent resistance is explained by a dominant role of the electron-electron scattering. However, in our case this explanation is unusable. The insertion of GBs in the polycrystalline films leads to enhancement of the impurity (elastic) contribution to the resistance, only, and all inelastic scattering processes have to 
become weakly defined on the temperature dependence of the resistance. We assume that the $T^{2}$ contribution to the resistance can be attributed to the interference between the elastic electron scattering on GBs and the electron-magnon scattering, similar to what was observed in disordered metal films with domination of the electron-phonon scattering [27].

Figure $6, b$ displays the $\ln (R)$ versus $T^{-1 / 2}$ plot for both PCML films. This plot exhibits a linear dependence up to $T_{\min }$ and manifests the exponential growth of resistance at low temperature, which is described by the expression $R(T) \propto \exp \sqrt{\Delta / T}$. It is noteworthy that a similar expression with $\Delta \propto E_{C}$, where $E_{C}$ is the charging energy, has been predicted for conductivity in granular metals [28] and used for the explanation of the low-temperature $R(T)$ behavior in ceramic $\mathrm{La}_{2}{ }_{3} \mathrm{Sr}_{1 / 3} \mathrm{MnO}_{3}$ manganite with different grain size [25]. The value of the charging energy estimated from the slope of the $\ln (R)$ versus $T^{-1 / 2}$ plot was $E_{C} \simeq 20 \mathrm{~K}$ and $\simeq 2.46 \mathrm{~K}$ for the PCML1 and PCML2 films, respectively. Our results are very close to those obtained for ceramic samples [25], and the observed difference in $E_{C}$ value for different PCML films can be explained by the variation of the grain size. Indeed, the deposition of the manganite films at a low substrate temperature leads, as a rule, to the formation of a fine-grain structure, and an increase of $T_{\text {sub }}$ stimulates grain growth. On the other hand, $E_{C} \propto e^{2} / d$, where $e$ is the electronic charge and $d$ is the grain size [28]. Therefore, the observed larger $E_{C}$ value for the low- $T_{\text {sub }}$ PCML film with respect to the high- $T_{\text {sub }}$ one is an absolutely expected result.

Taking into account the peculiarities in $R(T)$ behavior which are governed by the existence of GBs, we will be again try to describe the temperature-dependent resistance of the PCML films on the basis of the parallel circuit model, only in this case the total resistance of the LSMO layers must involve the sum of $R_{\mathrm{LSMO}}+R_{\mathrm{GBs}}$. Figure 7 shows that in the framework of this approach the nonmonotonic $R(T)$ behavior can be described with satisfactory accuracy (solid lines in Fig. 7). We don't present here the fitting parameters used because of their multiplicity and the difficulty in interpretation of the physical meaning. However, the carried out analysis allows us to conclude that observed peaks in $R(T)$ are not provided by the Gbs [8] but come out from the parallel-resistance circuit of the LSMO and PCMO layers.

The negative MR of the polycrystalline manganites is dominated by spin-polarized tunneling between grains owing to a nearly complete polarization of the electrons [6]. The simplified equation for the MR in the framework of the spin-polarized tunneling model $[29,30]$ can be written as

$$
\mathrm{MR} \simeq-\frac{J P}{4 k_{B} T}\left[m^{2}(H, T)-m^{2}(0, T)\right],
$$

where $J$ is an intergrain exchange constant, $P$ is the electron polarization, and $m$ is the magnetization normalized to the saturation value. The inset in Fig. 7 shows the dependence of the MR versus $m^{2}$ plot, where $m=M(T) / M(0)$ and $M(T)$ is the FC magnetization of the PCML films presented in Fig. 4,b. It is seen that the MR ratio is approximately $\propto m^{2}$ for the both polycrystalline films. On the other hand, the temperature dependence of the MR ratio (see inset in Fig. 3) is very far from that predicted by this model, MR $\propto 1 / T$. This disagreement can be eliminated by considering the temperature dependence of the spin polarization calculated within the framework of the DE approach (see Fig. 1 in Ref. 24).

\section{Conclusions}

Summarizing, we have studied the magnetic and transport properties of single-crystal and poly-

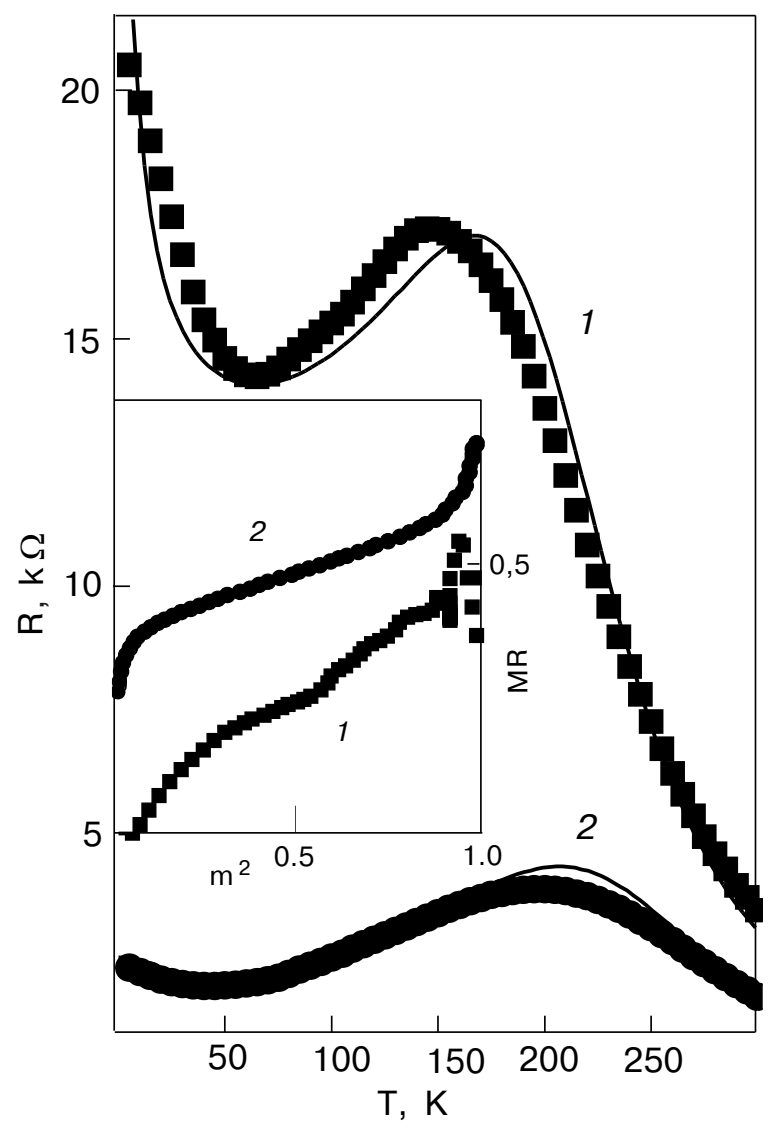

Fig. 7. The comparison of the experimental $R(T)$ data (solid symbols) with the theoretical simulation (solid lines) in the framework of the two parallel resistance model for the PCML1 (1) and the PCML2 (2) films, respectively. Inset displays the MR ratio dependence on the square of the normalized magnetization for these films. 
crystalline PCMO/LSMO multilayered films. It was shown that the single-crystal ML film with an incoherent interface between layers manifests the magnetic properties typical for a two-phase magnetic system. The temperature-dependent resistance of this film in the ferromagnetic metallic state is proportional to $T^{3}$ and has been attributed to the one-magnon-electron scattering. The formation of a coherent interface between layers, owing to the enhancement of the epitaxial growth mode, leads to the origin of the ferromagnetic coupling between layers and to the domination of a $T^{4.5}$ term in $R(T)$, which is typical for the two-magnon-electron scattering. The negative MR of the single-crystal ML films, which reaches almost $60 \%$ at room temperature in an applied magnetic field of $5 \mathrm{~T}$, is provided by the MI transition in the LSMO layer near the Curie temperature. The polycrystalline ML films demonstrate the $R \propto T^{2}$ behavior, which is explained by the interference between the elastic electron scattering on GBs and the inelastic electron-magnon scattering. The enhancement of the resistance at low temperature, $R(T) \propto \exp \sqrt{E_{C} / T}$, has its origin in a small Coulomb barrier which forms on the GBs. The negative MR of the polycrystalline ML films is dominated by a spin-polarized tunneling between grains and can be described within the framework of the DE model. The model of two parallel resistances can be used for simulation of the transport properties in the ML films.

This work was supported by the KOSEF through the Quantum Photonic Science Research Center.

1. Y. Tokura and Y. Tomioka, J. Magn. Magn. Mater. 200, 1 (1999).

2. A.P. Raminez, J. Phys. C9, 8171 (1997).

3. C. Zener, Phys. Rev. 82, 403 (1951).

4. P.W. Anderson and H. Hasegawa, Phys. Rev. 100, 675 (1955).

5. P.-G. de Gennes, Phys. Rev. 118, 141 (1960).

6. H.Y. Hwang, S-W. Cheong, N.P. Ong, and B. Batlogg, Phys. Rev. Lett. 77, 2041 (1996).

7. A. Gupta, G.Q. Gong, G. Xiao, P.R. Duncombe, P. Lecoeur, P. Trouilloud, Y.Y. Wang, V.P. Dravid, and J.Z. Sun, Phys. Rev. B54, R15629 (1996).

8. R. Gross, L. Alff, B. Büchner, B.H. Freitag, C. Höfener, J. Klein, Y. Lu, W. Mader, J.B. Philipp, M.S.R. Rao, P. Reutler, S. Ritter, S. Thienhaus, S. Uhlenbruck, and B. Wiedenhorst, J. Magn. Magn. Mater. 211, 150 (2000).
9. Y. Tomioka, A. Asamitsu, H. Kuwahara, Y. Morimoto, and Y. Tokura, Phys. Rev. B53, R1689 (1996).

10. V.G. Prokhorov, G.G. Kaminsky, V.S. Flis, Y.P. Lee, K.W. Kim, and I.I. Kravchenko, Physica B307, 239 (2001).

11. M.C. Martin, G. Shirane, Y. Endoh, K. Hirota, Y. Morimoto, and Y. Tokura, Phys. Rev. B53, 14285 (1996).

12. T. Wu, S.B. Ogale, S.R. Shinde, Amian Biswas, T. Polletto, R.L. Greene, T. Venkatesan, and A.J. Millis, J. Appl. Phys. 93, 5507 (2003).

13. I. Medvedeva, A. Maignan, K.Bärner, Yu. Bersenev, A. Roev, and B. Reveau, Physica B325, 57 (2003).

14. Z. Jirák, S. Krupička, Z. Šimša, M. Dlouhá, and S. Vratislav, J. Magn. Magn. Mater. 53, 153 (1985).

15. F. Tsui, M.C. Smoak, T.K. Nath, and C.B. Eom, Appl. Phys. Lett. 76, 2421 (2000).

16. M. Sirena, N. Haberkom, M. Granada, L.B. Steren, and J. Guimpel, J. Appl. Phys. 93, 7244 (2003).

17. H. Li, J.R. Sun, and H.K. Wong, Appl. Phys. Lett. 80, 628 (2002).

18. Y. Sun, W. Tong, X. Xu, and Y. Zhang, Appl. Phys. Lett. 78, 643 (2001).

19. E. Dagotto, T. Hotta, and A. Moreo, Phys. Rep. 344, 1 (2001).

20. M. Sirena, N. Haberkom, L.B. Steren, and J. Guimpel, J. Appl. Phys. 93, 6177 (2003).

21. M. Imada, A. Fujimori, and Y. Tokura, Rev. Mod. Phys. 70, 1039 (1998).

22. N. Furukawa, Y. Shimomura, T. Akimoto, and Y. Moritomo, J. Magn. Magn. Mater. 226-230, 782 (2001).

23. K. Kubo and N. Ohata, J. Phys. Soc. Jpn. 33, 21 (1972)

24. H. Itoh, T. Ohsawa, and J. Inoue, Phys. Rev. Lett. 84, 2501 (2000).

25. Lí. Balcells, J. Fontcuberta, B. Martínez, and X. Obradors, Phys. Rev. B58, R14697 (1998).

26. J.M.D. Coey, M. Viret, and S. von Molnar, Adv. Phys. 48, 167 (1999).

27. N.G. Ptisina, G.M. Chulkova, K.S. Il'in, A.V. Sergeev, F.S. Pochinkov, E.M. Gershenzon, and M.E. Gershenzon, Phys. Rev. B56, 10089 (1997).

28. P. Sheng, B. Abeles, and Y. Arie, Phys. Rev. Lett. 31, 44 (1973).

29. J.I. Gittleman, Y. Goldstein, and S. Bozowski, Phys. Rev. B5, 3609 (1972).

30. J.S. Helman and B. Abeles, Phys. Rev. Lett. 37, 1429 (1976). 